\title{
Focus on bioanalysis
}

\author{
Antje J. Baeumner • Günter Gauglitz • \\ Frieder W. Scheller
}

Published online: 21 September 2010

(C) Springer-Verlag 2010

Analytical and Bioanalytical Chemistry was established in 2002 through the merger of Fresenius' Journal of Analytical Chemistry, Analusis, and Química Analítica. The merger reflected not only the internationalization of the subject but also a shift in focus towards bioanalysis. Accordingly, two special issues were published in 2003 and 2008 highlighting the vibrant area of bioanalysis. The success of these special issues encouraged the Editorial Board of Analytical and Bioanalytical Chemistry to schedule a further special issue on this topic. Antje Baeumner has now joined, as a guest editor, in inviting the scientific community to submit original papers and reviews on the state-of-the-art of bioanalysis. Today the bioanalytical focus of the journal features both:

- the development and application of analytical methods for the life sciences, biology, and medicine; and

- the creation and adaptation of biological molecules and systems for analytical applications.

Published in the special issue Focus on Bioanalysis with Guest Editors Antje J. Baeumner, Günter Gauglitz, and Frieder W. Scheller.

\footnotetext{
A. J. Baeumner

Department of Biological and Environmental Engineering,

Cornell University,

Ithaca, NY 14853, USA

e-mail: Baeumner@cornell.edu

G. Gauglitz $(\square)$

Institut für Physikalische und Theoretische Chemie,

Auf der Morgenstelle 8,

72076 Tübingen, Germany

e-mail: guenter.gauglitz@ipc.uni-tuebingen.de

F. W. Scheller

Mathematisch-Naturwissenschaftliche Fakultät, Institut für

Biochemie und Biologie, Universität Potsdam,

Karl-Liebknecht-Straße 24-25, Haus 25,

14476 Golm, Germany

e-mail: fschell@uni-potsdam.de
}

Along these lines, this special issue has three main aims:

- to update readers on developments in the implementation of analytical methods;

- to provide a limited survey on modern applications; and - to offer some visions and trends for the future of bioanalysis.

The guest editors are well aware that this wide field cannot be covered in a single journal issue by trying to gather together the research of the many groups all over the world. However, in this issue the range of papers encompasses instrumental analysis, tools for nanobiotechnology, new recognition elements, and different types of assay. In review articles miniaturization of assays is discussed, e.g. for flow cytometry, microarrays, separation techniques, and elemental mass spectrometry. Nanobiotechnology applications, for example quantum dots and noble metal nanorods, are also reviewed. Bioanalysis for metabolomics, point-of-care diagnostics, bioprocess monitoring, non-invasive cell-based sensing, DNA-protein interactions, and emerging food contaminants are presented. Reviews on direct optical detection, label-free analysis, solid-state NMR, LC-MS-MS and new recognition elements including aptamers, and molecularly imprinted polymers (MIPs) are also included. Similarly, original papers cover an equally wide range including aptamer-based assays, SPR, microelectrochemical systems, microtable arrays, ELISAs, and MALDI-TOF MS.

Thus, the special issue provides both a status report on current developments and an outlook on future trends in upcoming fields; it thus complements the numerous other special issues in $\mathrm{ABC}$ which have been devoted to selected fields of applications of (bio)analytical methods, e.g. forensic analysis, marine ecotoxicology, diagnostic assays, allergens in foods, antibiotics in food and the environment, mycotoxins, phytotoxins and cyanotoxins, and GMOs. 
Further special issues have recently featured not only methods and tools of instrumental analysis, e.g. mass spectroscopy, optical sensing, bioanalytical luminescence, quantum dots for luminescence, analytical separations, tools for the nanoworld, but also biological recognition, e.g. analysis of cytochrome P-450 and RNA analysis. Looking at the special issues highlighting analytical research in different countries - such as that published on Germany in 2009-it becomes very apparent that in recent years the interest, development, and application of bioanalysis have increased tremendously in comparison with the former focus on chemical analysis. The use of the classical methods of separation techniques, spectroscopy, and spectrometry in bioanalysis require only small adaptation to be applicable in many fields, even in "omics".

The use of biological molecules and systems as recognition elements permits new applications such as in process analysis (not only in biotechnology), going to very low concentrations in environmental samples; measuring, without sample preparation, in specimens such as milk, meat, or blood; and using artificial membranes and pores and nanoparticles with imprinting. Drastic signal enhancement will be enabled by taking advantage of dendrimer-like structures and using tip-enhanced Raman spectroscopy.

The use of imaging and biophotonics in bioanalysis is another important new application fields, such as approaches that label of carrier molecules which will pass the membrane barrier into the cell. In fact, subsequent single-molecule detection or near-field optics are a prerequisite for understanding biological and medical incidents, and in the future will be one of the requirements of system biology for the study of signalling chains. Especially the new methods in near-field optics will bring research in medicine an enormous step forward.

It is important to realize how essential good analysis is in many of these new areas. For instance, imaging techniques will make it possible to overcome stochastic problems by looking at single molecules. In the biophotonics area analysts need to contribute their knowledge in order to achieve results that are quantitatively reliable. Also, challenges associated with nano- or micro-based analytical systems are plentiful and go beyond successful design, fabrication, testing, and statistics. The need for quality assurance as related to LODs, LOQs, and plausibility control is especially apparent as collaborations with biologists, physicians, and biochemists increase. This is valid also for another upcoming field-point-of-care testing and other evolutions in clinical diagnostics. Miniaturization, parallelization, and imaging are important topics in this issue of the journal, reflecting the intense research in these areas, supported by calls from numerous funding agencies such as the European Commission, the National Science Foundation, and the National Institutes of Health, to name but a few.

Numerous challenges in modern analytical and, especially, bioanalytical research lie ahead of us. ABC feels not only well prepared to inform the scientific community and to supply outstanding progress reports, but also to reveal limitations and kinks in the solutions of scientific problems that need to be addressed.

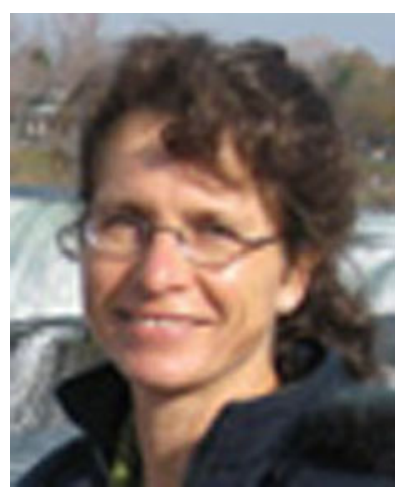

Antje J. Baeumner is Professor Biological Engineering and Director of Graduate Studies in the Department of Biological and Environmental Engineering at Cornell University in Ithaca, NY, USA. She is on the Board of Directors of the Society of Electroanalytical Chemistry, a member of the Extended Executive Committee of the International Association of Environmental Analytical Chemistry, was the 2010 Chair of the Gordon Research Conference on Bioanalytical Sensors, and has received numerous honors in recent years including being Finalist for the Blavatnik Award of the NY Academy of Sciences, and receiving a Humboldt Research Fellowship and a German National Science Foundation Mercator Guest professorship. Her research is focused on the development of microTotal Analysis Systems and smart lateral flow assays for the detection of pathogens and toxins in food, in the environment, and for medical diagnostics.

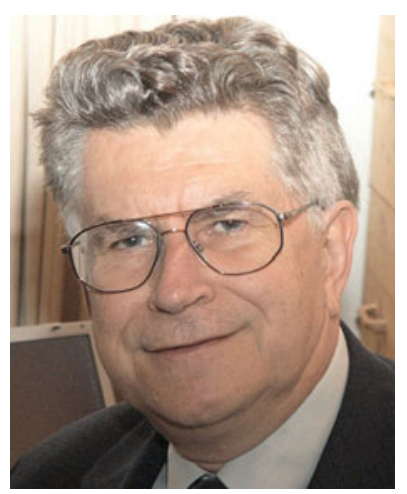

Günter Gauglitz is Professor at the Eberhard-Karls-University of Tübingen working in analytical and physical chemistry. He is chairman of the GDCh Division of Analytical Chemistry and chaired the Europt(r)ode VIII meeting. For the last 10 years his main scientific interests have centered on research and development in chemical and biochemical sensors with special focus on the characterization of interfaces of polymers and biomembrane surfaces, spectroscopic techniques, use of spectral interferometry to monitor changes in optical thickness of thin layers, and effects of Fresnel reflectivity at interfaces. He has been Editor of Analytical and Bioanalytical Chemistry $(A B C)$ since 2002. 


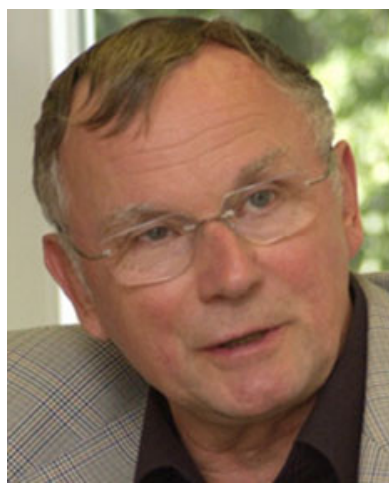

Frieder W. Scheller is visiting Professor at the University of Potsdam and consultant at the Fraunhofer Institute for Biomedical Engineering. For many years he headed the Department of Bioelectrochemistry/Biosensors at the Central Institute of Molecular Biology of the German Academy of Sciences, Berlin-Buch. He has been Professor of Analytical Biochemistry at the University of Potsdam since 1993. In 2001 he was President of the German Society of Biochemistry and Molecular Biology. His current research interests include biomimetic sensors based on molecularly imprinted polymers and protein electrochemistry. 